\title{
Mobility impairments and pelvic health disorders in women: the need for innovative treatment and research
}

\author{
Sophie Fletcher • Margaret A. Nosek • Diaa E. E. Rizk
}

Received: 30 December 2013 / Accepted: 29 April 2014 / Published online: 14 June 2014

(C) The International Urogynecological Association 2014

Keywords Holistic care · Mobility impairments ·

Neurological diseases $\cdot$ Pelvic health $\cdot$ Women

"I've had a disability for 45 years and I'm dealing with it just fine. But what's with all this new stuff, all these aches and pains? Breathing is getting worse, circulation is getting worse, my bladder is giving me problems now. I'm too young to be feeling so old!" 45 -year-old woman with a neuromuscular disorder [1]

Nearly 12 million or $8 \%$ of civilian, non-institutionalized women in the United States suffer from mobility impairment [2], a subpopulation that is increasing in size with the aging of the general population and improved survival rates from disease and injury. Mobility impairment is a functional limitation affecting ambulation that results from a primary disorder of the neuromuscular, skeletal, or central nervous system or of the joint and connective tissues. It is often associated with physical and psychological co-morbidities, referred to in

\footnotetext{
S. Fletcher $(\bowtie)$

Department of Urology, Kaiser Permanente, 401 Bicentennial Way,

Santa Rosa, CA 95403, USA

e-mail: sophiegfletcher@gmail.com

\section{A. Nosek}

Department of Physical Medicine and Rehabilitation, Baylor College of Medicine, Houston, TX, USA

\section{E. E. Rizk}

Department of Obstetrics and Gynecology, Faculty of Medicine, Ain Shams University, Cairo, Egypt

D. E. E. Rizk

Egypt and The Canadian Continence Foundation, Peterborough, ON, Canada
}

rehabilitation research as secondary conditions. The most common secondary conditions are obesity [3], hypertension, pain, skin breakdown, type II diabetes mellitus, digestive problems, and elimination difficulties including bowel and bladder problems such as urge incontinence [4]. Pelvic health in women has a wider definition than appropriate urinary and fecal control, integrity of pelvic organ support, and normal sexual function. A broader clinical outlook takes into consideration the adverse impact that associated disabilities and co-morbidities has on women's pelvic health. One example is that of the screening, detection, and management of diabetes mellitus and impaired renal function respectively, to prevent renal insufficiency and metabolic disturbances in women with pelvic health disorders. This has been the subject of previous editorials in the Journal $[5,6]$

When disorders such as spinal cord injury (SCI), multiple sclerosis (MS) or stroke affect control of one component of the pelvis, others are likely to be dysfunctional as well. Muscle spasticity in one muscle group can lead to a combination of constipation, urinary retention, and sexual pain in the same patient. Leakage of urine and/or stool puts patients at risk of urinary tract infections, dermatitis, and skin breakdown, not to mention embarrassment, depression, and avoidance of intimate and social interaction. Limitations of mobility, particularly in women, further exacerbate these problems as the female urethra and vagina are particularly susceptible to infection in the immobile pelvis [1, 4]. For these reasons, optimal care for disabled women complaining of dysfunction in one pelvic organ system necessitates screening for symptoms in the others.

It is well documented that women with disabilities tend to receive inadequate gynecological screening and care. These patients are less likely than non-disabled women to be referred for cervical cancer screening and mammograms. Breast 
cancer in disabled women is diagnosed in its more advanced stages, and breast cancer mortality rates are higher compared with women without disabilities. At some point in their adult life, $31 \%$ of women with disabilities have been refused care by a physician [1]. Similar disparities may exist regarding urogynecological screening and care in this population. With the growth of aging female populations around the world, the field of female pelvic medicine is expanding exponentially. Now more than ever is the time for specialists in this area to examine the physical barriers and lack of information regarding the special pelvic floor concerns of disabled women.

An assumption is made sometimes that inactive women do not experience pelvic organ descent. In our referral center, we see many physically disabled women with symptomatic POP. Women with disabilities of mobility, even those with extensive limitations, have pregnancies with vaginal deliveries and others may experience their neurological disorder after childbearing. Regardless of the disability, POP does occur, and management requires a specialist familiar with the urinary incontinence, urinary retention, chronic urinary tract infections, chronic constipation, and surgical recovery challenges that these patients experience. Unfortunately, lack of research into POP in this population results in a paucity of knowledge on the subject, and more frustration for clinicians seeking guidance from the literature.

"I always felt like a neutral sex. It's like I'm not a woman, not a man. I don't know what I am because I was never approached like a woman and I guess that as I grow older and mature more, I have begun to proclaim that identity as a woman and thinking even if no man approached me, I am still a woman, I am still attractive.”

36-year-old polio survivor with scoliosis [1]

Women with impairments of mobility are sexually active and sexual health is an important quality of life component throughout age ranges. Up to $80 \%$ of adult women with SCI are sexually active, and depending on the level of injury $59 \%$ can achieve orgasm [7]. Sexual health impairment has a substantial impact on quality of life for women with MS, even after adjusting for disability status and age [8]. Sexual dysfunction is likely under-diagnosed in these populations, probably because the topic is rarely approached by physicians. In fact, studies have shown that the majority of women with SCI are dissatisfied with the amount and quality of information about sexual health provided by their physician [7]. Other populations such as women with cerebral palsy or joint and connective tissue diseases have also expressed similar frustrations [1]. If we don't ask these women about their sex lives, who will?
Many of the secondary conditions contributing to female sexual dysfunction in this population are well understood by female pelvic medicine specialists. Mobility impairment is a risk factor for bone loss that must be taken into account with hormone replacement therapy for vaginal atrophy, menopause, and sexual dysfunction. When spasticity or neuropathic pain plays a role in sexual dysfunction, collaboration with neurologists and physical medicine and rehabilitation colleagues provides excellent multidisciplinary solutions [9].

At present, there is an increasing need for state-ofthe-art female pelvic health care in the context of impaired mobility. Research and clinical guidelines on the holistic care of women with pelvic health problems and physical disabilities, however, is lacking. Investigator initiatives should focus on models for improvement, but in many instances, it is not even clear what "improvement" means for these patients. The development and implementation of such models are perfectly suited to the field of female pelvic medicine. As the topic grows, it would be a venerable feat to close this disparity gap early and add another layer of prowess to our field.

\section{References}

1. Nosek MA, Howland CA, Rintala DH, Young ME, Chanpong GF (2001) National Study of Women with Physical Disabilities: final report. Sex Disab 19:5-39

2. US Census Bureau (2013) B18105: Sex by age by ambulatory difficulty. Universe: Civilian noninstitutionalized population 5 years and over; 2011 American Community Survey 1-year estimates. US Census Bureau, Washington DC

3. Nosek MA, Robinson-Whelen S, Hughes RB et al (2008) Overweight and obesity in women with physical disabilities: associations with demographic and disability characteristics and secondary conditions. Disabil Health J 1:89-98

4. Nosek MA, Hughes RB, Petersen NJ et al (2006) Secondary conditions in a community-based sample of women with physical disabilities over a 1-year period. Arch Phys Med Rehabil 87:320-327

5. Rizk DEE, Obineche EN (2007) Female pelvic floor disorders and impaired renal function: an appraisal. Int Urogynecol J 18:1253-1255

6. Abdel-Fattah M, Rizk DEE (2012) Diabetes mellitus and female urinary incontinence: a time for change. Int Urogynecol J 23:14811482

7. Kreuter M, Taft C, Siösteen A, Biering-Sørensen F (2011) Women's sexual functioning and sex life after spinal cord injury. Spinal Cord 49: 154-160

8. Nortvedt M, Riise T, Myhr K, Landtblom A, Bakke A, Nyland H (2001) Reduced quality of life among multiple sclerosis patients with sexual disturbance and bladder dysfunction. Mult Scler 7:231-235

9. Fletcher SG, Haverkorn RM, Yan J, Lee JJ, Zimmern PE, Lemack GE (2010) Demographic and urodynamic factors associated with persistent $\mathrm{OAB}$ after anterior compartment prolapse repair. Neurourol Urodynam 29:1414-1418 